\title{
Dexamethasone Dipropionate Loaded Nanoparticles of $\alpha$-Elastin-g- PLGA for Potential Treatment of Restenosis
}

\author{
Fabio S. Palumbo, ${ }^{\dagger}$ Salvatrice Rigogliuso, ${ }^{\S}$ Giulio Ghersi, ${ }^{\S}$ Giovanna Pitarresi, ${ }^{*}, \dagger, \|$ Fiorica Calogero, ${ }^{\dagger}$ \\ Mauro Di Stefano, ${ }^{\dagger}$ and Gaetano Giammona ${ }^{\dagger, \ddagger}, \|$
${ }^{\dagger}$ Dipartimento di Scienze e Tecnologie Biologiche Chimiche e Farmaceutiche, Plesso di Chimica e Tecnologie Farmaceutiche, Università degli Studi di Palermo, Via Archirafi 32, 90123 Palermo, Italy
${ }^{\ddagger}$ IBIM-CNR, Via Ugo La Malfa 153, 90146 Palermo, Italy
${ }^{\S}$ Dipartimento di Scienze e Tecnologie Biologiche Chimiche e Farmaceutiche, Plesso di Biologia Cellulare, Università degli Studi di Palermo, Viale delle Scienze ed. 16, 90128 Palermo, Italy
"Institute of Biophysics at Palermo, Italian National Research Council, Via Ugo La Malfa 153, 90146 Palermo, Italy

\begin{abstract}
A graft copolymer of $\alpha$-elastin with poly(lactic-co-glycolic) acid (PLGA) has been synthesized and successfully employed to produce nanoparticles. Exploiting the known biological activity of $\alpha$-elastin to promote the maintenance of smooth muscle cells (SMCs) contractile phenotype and the antiproliferative effect of glucocorticoids, the aim of this research was to produce drug-loaded nanoparticles suitable for potential treatment of restenosis. In particular, nanoparticles of $\alpha$-elasting-PLGA with a mean size of $200 \mathrm{~nm}$ have been produced and loaded with dexamethasone dipropionate $(10 \% \mathrm{w} / \mathrm{w})$, chosen as a model drug that inhibits proliferation of vascular SMCs. These nanoparticles are able to prolong the drug release and show a pronounced sensibility to elastase. Drug unloaded nanoparticles stimulate the differentiation of human umbilical artery smooth muscle cells (HUASMCs) toward the contractile
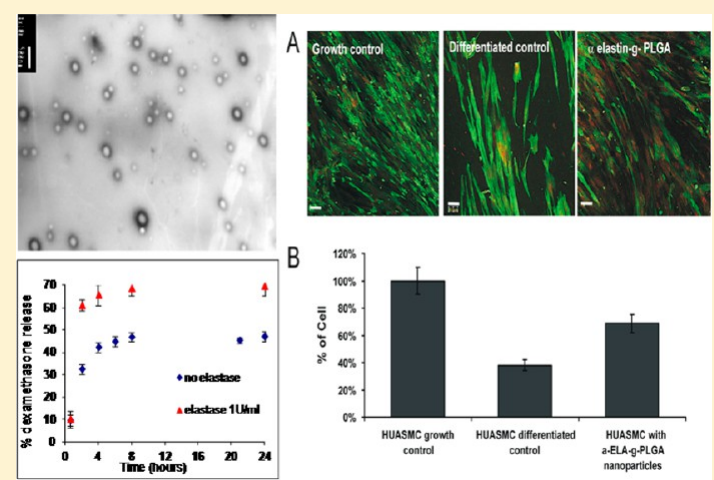
phenotype as demonstrated by immunofluorescence, flow cytofluorimetric, and western blotting analyses. Finally, drug-loaded nanoparticles efficiently reduce viability of HUASMCs as evidenced by 3-(4,5-dimethylthiazol-2-yl)-5-(3-carboxymethox-
\end{abstract} yphenyl)-2- (4-sulfophenyl)-2H-tetrazolium, inner salt (MTS) assay.

KEYWORDS: $\alpha$-elastin, poly(lactic-co-glycolic) acid, graft copolymer, nanoparticles, restenosis, dexamethasone dipropionate

\section{INTRODUCTION}

Vascular healing is a complex physiological process essential for maintaining vessel integrity and functionality. A dysfunction of this process can produce an intimal hyperplasia because of an abnormal migration and proliferation of vascular smooth muscle cells (VSMCs). ${ }^{1}$ This hyperplasia can produce a phenomenon known as restenosis, that leads to the vessel obstruction where a tissue engineered vascular graft (TEVG) or a stent has been implanted. Thus, the inhibition of this hyperplasia is important to prolong patency of the implanted TEGV or stent functionality. At this aim, the specific target of therapeutic treatment is the key event of the hyperplasia, i.e., the proliferation of VSMCs. Indeed, VSMCs can assume both a proliferative and contractile phenotype; ${ }^{2}$ in the first case, cells can rapidly proliferate and produce an extracellular matrix, while the change into a contractile phenotype would allow a better performance of TEGV or stent, thus reducing the risk of restenosis. Several drugs, growth factors, or extracellular matrix stimulating factors are potentially able to control the differentiation of VSMCs inhibiting the proliferative phenotype, and some of these agents are currently in therapeutic use. ${ }^{3,4}$ In particular, several antiinflammatory drugs are employed to reduce proliferation of VSMCs after a reconstructive surgery or they are loaded into stents to prevent restenosis. ${ }^{5,6}$ However, the administration or loading of these drugs into stents without an appropriate carrier able to prolong their release makes such treatments often unsuccessful. ${ }^{7,8}$

Elastin is known to maintain a contractile phenotype of VSMCs, ${ }^{9,10}$ inhibiting their migration and proliferation. ${ }^{1}$ It has a morphogenetic role on differentiaton of SMC, acting via a Gprotein-coupled signaling pathway, ${ }^{1}$ and it has demonstrated a reduction of restenosis after its administration in a pig model. Dexamethasone dipropionate inhibits proliferation of VSMCs, ${ }^{11}$ blocking cell cycle progression late in the G1 phase, and it has already been employed to treat restenosis in stenting ${ }^{3}$ both as free drug and loaded into polymeric nanoparticles. ${ }^{12}$

It is evident that, even if through a different mechanism, both elastin and dexamethasone are potentially employable to reduce

Received: July 17, 2013

Revised: October 3, 2013

Accepted: November 8, 2013

Published: November 8, 2013 
restenosis and then if administered together they could have an interesting synergetic effect allowing to act both on SMC phenotype and directly on differentiated cells, inhibiting their proliferation. For this reason, in this work, we have prepared new nanoparticles based on an $\alpha$-elastin grafted to a biocompatible and biodegradable polyester, such as polylacticco-glycolic acid (PLGA), loaded with dexamethasone dipropionate. Physicochemical and biological properties of these nanoparticles have been evaluated, as well as their ability to prolong drug release.

\section{EXPERIMENTAL SECTION}

Materials and Methods. Poly(lactic-co-glycolic) acid (PLGA) Resomer RG $502 \mathrm{H}$ with an inherent viscosity of $0.19 \mathrm{dL} / \mathrm{g}, M_{\mathrm{w}} 3000 \mathrm{Da}$, was purchased by BoehringerIngelheim (Milan, Italy). Bis(4-nitrophenyl)carbonate (4NPBC), 4-nitrophenyl chloroformate, elastin from bovine neck ligament, pancreatic elastase from porcine pancreas, pyridine, dexamethasone dipropionate (DEX), Dulbecco's phosphate buffered solution (DPBS), and Dulbecco's modified eagle medium (DMEM) were purchased from Sigma-Aldrich (Milan, Italy). D-(+) trealose dihydrate and ethylenediamine (EDA) were purchased from Fluka (Milan, Italy).

FT-IR spectra were performed by using a Perkin-Elmer 1720 spectrophotometer in the range $4000-400 \mathrm{~cm}^{-1}$. ${ }^{1} \mathrm{H}$ NMR spectra were obtained with a Bruker AC-300 instrument. High performance liquid chromatography (HPLC) analysis has been performed by using an Agilent 1100 series with an Agilent injector $7725 \mathrm{i}$ and a UV detector Agilent 1100 equipped with a column Symmetry (Waters, C18, $5 \mu \mathrm{m} 150 \times 4.6 \mathrm{~mm}$ ), employing as an eluent acetate buffer $1 \mathrm{mM} \mathrm{pH} 4.8$ /acetonitrile in a ratio $25 / 75 \mathrm{v} / \mathrm{v}$, with a flow of $0.8 \mathrm{~mL} / \mathrm{min}$ and a $\lambda$ value of $243 \mathrm{~nm}$. Size exclusion chromatography (SEC) analysis was performed using a multidetector SEC system equipped with a Water 600 pump and a Water 410 Refractive Index Detector with Phenogel column employing as an eluent $\mathrm{LiCl} 0.05 \mathrm{M}$ in DMF mixed with DMSO in a ratio 9:1 and a flow of $0.6 \mathrm{~mL} /$ min. Dynamic light scattering measurements were performed by using a Zetasizer Nano ZS instrument (Malvern Instrument, Germany). Transmission electron microscopy (TEM) images were recorded by using a JEOL JEM-2100 instrument.

$\boldsymbol{\alpha}$-Elastin Extraction from Bovine Elastin. The extraction of $\alpha$-elastin from elastin of bovine neck was performed following the procedure of Partridge et al. ${ }^{13}$ Briefly, $1 \mathrm{~g}$ of bovine elastin was suspended in $0.25 \mathrm{M}$ oxalic acid solution and hydrolyzed by sequential cycles of heating for $1 \mathrm{~h}$ in a water boiling bath. The supernatant was collected by centrifugation, and the precipitated solid was treated until complete hydrolysis. The collected soluble fractions were dialyzed against water using a dialysis membrane tube of $3500 \mathrm{Da}$ and then freezedried. The obtained powder was dissolved in acetic acid solution $0.01 \mathrm{M}$ containing $\mathrm{NaCl} 0.1 \mathrm{M}(1 \mathrm{~g}$ of hydrolyzed elastin in $10 \mathrm{~mL}$ of acidic solution) and heated at $50{ }^{\circ} \mathrm{C}$ for 15 min to obtain the $\alpha$-elastin coacervation. The coacervate was collected by centrifugation. The procedure was repeated until complete precipitation of $\alpha$-elastin. The $\alpha$-elastin was finally dissolved in acetic acid solution $0.1 \mathrm{M}$ and dialyzed against acetic acid solution using a dialysis membrane of $3500 \mathrm{Da}$ at 4 ${ }^{\circ} \mathrm{C}$ for 1 day. Finally, the solution was freeze-dried and the $\alpha$ elastin was stored as a powder.

Synthesis of PLGA-Ethylenediamine (PLGA-EDA) Derivative. The synthesis of the PLGA-EDA derivative was performed similarly to the functionalization of polycaprolac- tone, as described by Choi and colleagues, ${ }^{14}$ with some modifications. In particular, pyridine and 4-nitrophenyl chloroformate have been added to a solution of PLGA in dichloromethane $10 \% \mathrm{w} / \mathrm{v}$ in a molar excess of 1.2 and 3 , respectively. After $3 \mathrm{~h}$ at room temperature, the PLGAnitrophenyl derivative was precipitated into an excess of diethyl ether, washed with ethanol, and dried under a vacuum (yield $100 \% \mathrm{w} / \mathrm{w})$. The ${ }^{1} \mathrm{H}$ NMR spectrum $\left(\mathrm{CDCl}_{3}\right)$ showed the following peaks: $\delta 1.59\left(2 \mathrm{~d}, 3 \mathrm{H},-\mathrm{CO}-\mathrm{CH}\left(\mathbf{C H}_{3}\right)-\mathrm{O}-\right.$ of PLGA), $\delta 4.86$ (s, 2H, -CO- $\mathrm{CH}_{2}-\mathrm{O}-$ of PLGA), $\delta 5.22$ (2d, $1 \mathrm{H},-\mathrm{CO}-\mathrm{CH}\left(\mathrm{CH}_{3}\right)-\mathrm{O}-$ of PLGA), $\delta 8.03-\delta 8.87(\mathrm{~m}, 4 \mathrm{H}$, $p$-nitrophenyl moiety).

Ethylenediamine (EDA) was added to the solution of PLGAnitrophenyl derivative $(4 \% \mathrm{w} / \mathrm{v})$ in dichloromethane, in a molar ratio EDA/PLGA-nitrophenyl derivative equal to 2 . After $3 \mathrm{~h}$ at room temperature, the PLGA-EDA derivative was purified by precipitation into diethyl ether; then, the product was dissolved in acetone and precipitated into few volumes of $\mathrm{NaCl}$ saturated solution (100 $\mu \mathrm{L}$ per gram of product). Finally, the product was washed with a mixture ethanol/water $8: 2$ and then with ethanol. The purification of the PLGA-EDA derivative was confirmed by HPLC analysis. The ${ }^{1} \mathrm{H}$ NMR spectrum (in $\left.\mathrm{CDCl}_{3}\right)$ of PLGA-EDA derivative showed peaks at $\delta 1.59(2 \mathrm{~d}$, $3 \mathrm{H},-\mathrm{CO}-\mathrm{CH}\left(\mathbf{C H}_{3}\right)-\mathrm{O}-$ of PLGA), $\delta 2.2(\mathrm{~m}, 2 \mathrm{H},-\mathrm{CO}-$ $\mathrm{NH}-\mathrm{CH}_{2}-\mathrm{CH}_{2}-\mathrm{NH}_{2}$ of EDA), $\delta 4.85$ (s, $2 \mathrm{H},-\mathrm{CO}-\mathrm{CH}_{2}-$ $\mathrm{O}-$ of PLGA), and $\delta 5.21\left(2 \mathrm{~d}, 1 \mathrm{H},-\mathrm{CO}-\mathrm{CH}\left(\mathrm{CH}_{3}\right)-\mathrm{O}-\right.$ of PLGA).

Synthesis of $\boldsymbol{\alpha}$-Elastin-g-PLGA Copolymers. Three different weight ratios of PLGA-EDA/ $\alpha$-elastin were employed equal to 2, 4, and 6 (samples a, b, and c, respectively), and a two-step reaction was performed in the following way.

Step 1: $\alpha$-elastin $(3.3 \% \mathrm{w} / \mathrm{v}$ in anhydrous DMSO) was activated by employing 4-NPBC (weight ratio $\alpha$-elastin/4NPBC equal to 6 ) for $3 \mathrm{~h}$ at $40{ }^{\circ} \mathrm{C} .{ }^{15}$ Step 2: the solution of activated $\alpha$-elastin was added to PLGA-EDA solution ( $40 \% \mathrm{w} / \mathrm{v}$ in anhydrous DMSO) in an amount corresponding to the chosen weight ratio reported above. The reaction solution was maintained at $40{ }^{\circ} \mathrm{C}$ for $24 \mathrm{~h}$. Then, it was precipitated in ethanol and the product was washed with acetone. The $\alpha$ elastin-g-PLGA was obtained with a yield of $80 \% \mathrm{w} / \mathrm{w}$. Purification from the unreacted PLGA-EDA was confirmed by SEC analysis.

The FT-IR spectrum (in $\mathrm{KBr}$ ) showed the main following peaks: a broad band centered at $3450 \mathrm{~cm}^{-1}\left(\nu_{\text {as }} \mathrm{OH}+\nu_{\text {as }} \mathrm{NH}\right.$ of elastin), shoulder at $1780 \mathrm{~cm}^{-1}$ ( $\nu_{\text {as }}$ COO of PLGA), 1655 and $1533 \mathrm{~cm}^{-1}$ (amides of $\alpha$-elastin). The ${ }^{1} \mathrm{H}$ NMR spectrum (in DMF- $d_{6}$ ) showed the following peaks: $\delta 0.77$ and $1.22(\mathrm{~s}$, $9 \mathrm{H},-\mathrm{NH}-\mathrm{CH}\left(\mathrm{CH}_{3}\right) \mathrm{COO}-$ of alanine and $-\mathrm{NH}-\mathrm{CH}-$ $\left(\mathrm{CH}\left(\mathrm{CH}_{3}\right)_{2}\right)-\mathrm{COO}-$ of valine of $\alpha$-elastin, respectively), $\delta$ $1.59\left(2 \mathrm{~d}, 3 \mathrm{H},-\mathrm{CO}-\mathrm{CH}\left(\mathbf{C H}_{3}\right)-\mathrm{O}-\mathrm{PLGA}\right), \delta 4.9(\mathrm{~s}, 2 \mathrm{H}$, $\left.-\mathrm{CO}-\mathrm{CH}_{2}-\mathrm{O}-\mathrm{PLGA}\right)$, and $\delta 5.25(2 \mathrm{~d}, 1 \mathrm{H},-\mathrm{CO}-$ $\left.\mathrm{CH}\left(\mathrm{CH}_{3}\right)-\mathrm{O}-\mathrm{PLGA}\right)$. The amount of PLGA grafted to $\alpha$ elastin was calculated comparing the area of the peaks attributable to protons of $-\mathrm{CO}-\mathrm{CH}_{2}-\mathrm{O}-$ and $\mathrm{CO}-$ $\mathrm{CH}\left(\mathrm{CH}_{3}\right)-\mathrm{O}-$ of PLGA ( $\delta 4.9$ and 5.25 , respectively) with the area of peaks attributable to methyl groups of valine and alanine residues of $\alpha$-elastin.

In addition, to evaluate the activation efficiency of $\alpha$-elastin with $4-\mathrm{NPBC}$ in the first step of the reaction, the 4-NPBC activated $\alpha$-elastin was precipitated in acetone and washed several times with the same solvent. The molar amount of 4$\mathrm{NPBC}$ in $\alpha$-elastin was determined by colorimetric assay after treatment with $\mathrm{NaOH} 0.1 \mathrm{~N}$. In particular, $5 \mathrm{mg}$ of activated $\alpha$ - 
elastin was dissolved in $\mathrm{NaOH} 0.1 \mathrm{~N}$ and the absorbance was read at $405 \mathrm{~nm}$. A calibration curve of 4-NPBC in $\mathrm{NaOH} 0.1 \mathrm{~N}$ was used for the quantitative determination of 4-NPBC released from activated $\alpha$-elastin. The molar percentage of $\alpha$ elastin activated by 4-NPBC was determined considering a mean molecular weight of $105 \mathrm{Da}$ for aminoacids in $\alpha$-elastin as reported elsewhere. ${ }^{16}$

$\alpha$-Elastin-g-PLGA Nanoparticles: Preparation, Characterization, and in Vitro Drug Release Studies. $\alpha$-Elastin-gPLGA copolymer (sample a) was dispersed in DMSO with a concentration ranging from 2.5 to $15 \mathrm{mg} / \mathrm{mL}$. To prepare drugloaded nanoparticles, dexamethasone dipropionate was added in an amount corresponding to 0.5 -fold the weight of copolymer. Dispersions were added drop by drop with a microsyringe into an excess of bidistilled water (ratio DMSO solution:bidistilled water equal to $1: 15$ ) under stirring at 9500 rpm by using a Ultraturrax apparatus. The obtained dispersion was dialyzed against water for 3 days by using a Spectrapor dialysis tube (12000-14000 Da). Finally, a dialyzed dispersion was filtered through a $5 \mu \mathrm{m}$ cellulose acetate filter and freezedried. Size and zeta potential values of empty and drug-loaded nanoparticles were evaluated with a Zetasizer Nano ZS instrument in different media such as bidistilled water, DPBS $\mathrm{pH}$ 7.4, and DMEM. The size and shape of nanoparticles were also evaluated by TEM analysis. In particular, samples were prepared by dispersing $\alpha$-elastin-g-PLGA nanoparticles in bidistilled water $(1 \mathrm{mg} / \mathrm{mL})$ in a copper grid and drying at room temperature.

The amount of dexamethasone dipropionate loaded into nanoparticles was evaluated by HPLC analysis. In particular, 0.1 $\mathrm{mL}$ of a dispersion of drug-loaded nanoparticles in DMSO (10 $\mathrm{mg} / \mathrm{mL})$ was diluted with acetonitrile $(1 \mathrm{~mL})$ and then the obtained dispersion was filtered $(0.45 \mu \mathrm{m})$ before HPLC analysis. To evaluate the release of dexamethasone dipropionate, drug-loaded $\alpha$-elastin-g-PLGA nanoparticles were dispersed in DPBS pH 7.4 $(1 \mathrm{~mL})$ with or without the presence of elastase $(1 \mathrm{U} / \mathrm{mL})$ and sealed into a dialysis bag $(14000 \mathrm{Da})$ against $9 \mathrm{~mL}$ of DPBS $\mathrm{pH} 7.4$, under stirring and at $37^{\circ} \mathrm{C}$. At scheduled times, $1 \mathrm{~mL}$ of external medium was collected and used for drug quantification by HPLC and then replaced by fresh DPBS $\mathrm{pH}$ 7.4. After 1 and 10 days, nanoparticle dispersion was recovered from the dialysis bag and freezedried to determine the amount of dexamethasone dipropionate remaining in the nanoparticles. Each experiment was performed in triplicate.

In Vitro Culture of Human Umbilical Artery Smooth Muscle Cells. Human umbilical artery smooth muscle cells (HUASMCs) were purchased from European Collection of Cell Cultures (ECACC). Cells were cultured in a basal growth medium (Smooth Muscle Cell Growth Medium from Cell Application, Inc. Cat No $311 \mathrm{~K}-500)$ supplemented with 3\% growth factors, $10 \%$ fetal bovine serum (FBS; Euroclone Celbio), $1 \%$ antibiotic, and $1 \%$ glutamine (Euroclone Celbio). Cells were cultured in flasks $\left(75 \mathrm{~cm}^{2}\right.$; Corning), and when confluent, they were detached using tripsin-EDTA solution $1 \times$ (Sigma) and seeded on a culture multiwell plate (Corning) or on a coverslip, to perform different analyses.

Morphological Analysis. HUASMCs were seeded at low density ( 5000 cells/well) on a sterile coverslip in a culture 12 well-plate. After $24 \mathrm{~h}$, cells were incubated in a basal growth medium at $37^{\circ} \mathrm{C}$ alone (growth control) or in the presence of $30 \mu \mathrm{g} / \mathrm{mL} \alpha$-elastin-g-PLGA nanoparticles. As differentiated control, cells were incubated with a differentiating growth medium (Smooth Muscle Cell Differentiation Medium from Euroclone Celbio). This medium is specifically designed to promote differentiation of smooth muscle cells in culture and to maintain their mature contractile phenotype. The culture medium was changed every 2 days, and a new dose of nanoparticles $(30 \mu \mathrm{g} / \mathrm{mL})$ was added each time. After 4 days of incubation, cells were fixed on a coverslip using formaldehyde $3.7 \%$ for $15 \mathrm{~min}$ and permeabilized with Triton $0.1 \%$; then, the actin cytoskeleton was stained with phalloidin-FITC (1:500) for $1 \mathrm{~h}$ at $37^{\circ} \mathrm{C}$. Cells were thoroughly washed in phosphate buffer saline, and their nuclei were stained with propidium iodide $(1: 1000)$ for $1 \mathrm{~h}$ at $37^{\circ} \mathrm{C} .{ }^{17}$ Cell proliferation was evaluated by quantification of phalloidin-FITC actin fibers/field related to the mean cell area for each sample in the different culture conditions (10 random fields for each sample), using the Image J64 software. Data were expressed as arbitrary units (A.U.). Morphological analysis was performed by confocal microscopy observation (Olympus $1 \times 70$ with Melles Griot laser system).

Flow Cytofluorimetric Analysis. HUASMCs were seeded at high density (50000 cells/well) in a culture 6 well-plate, and after $24 \mathrm{~h}$, they were incubated as previously described. After 4 days of incubation, cells were detached using Tripsin-EDTA solution $1 \times$ and counted using the Thoma chamber, and then, 800000 cells/sample were centrifuged at $1000 \mathrm{rpm}$ for $10 \mathrm{~min}$. HUASMCs were treated with $500 \mu \mathrm{L}$ of propidium iodide solution $(50 \mu \mathrm{g} / \mathrm{mL})$ and incubated for $45 \mathrm{~min}$ under dark conditions. The cell cycle was analyzed by FACS (BD FACSDiva version 6.1.2). Each experiment was performed in triplicate.

Western Blotting Analysis. HUASMCs were seeded at high density (50000 cells/well) in a culture 6 well-plate, and after $24 \mathrm{~h}$, they were incubated as previously described. After 4 days of incubation, cells were mechanically detached and centrifuged at $1000 \mathrm{rpm}$ for $10 \mathrm{~min}$. The extracts were obtained treating the pelleted cells with $70 \mu \mathrm{L}$ of $1 \%$ Triton X-100 solution for $10 \mathrm{~min}$ at room temperature and then centrifuged at $10000 \mathrm{rpm}$ for $10 \mathrm{~min}$. The amount of proteins in the cell extracts was quantified by the Bradford microassay method (Bio-Rad, Segrate, Milan, Italy), using bovine serum albumin (Sigma-Aldrich) as a standard. A $5 \mu \mathrm{g}$ portion of each extract was analyzed by SDS-PAGE and blotted on nitrocellulose. By Western blotting analysis, the amount of $\alpha$ smooth muscle actin for each sample was evaluated and quantified by densitometric analysis with Image J64 software. Each experiment was performed in triplicate.

Cell Viability Assay. HUASMCs were seeded at high density (50 000 cells/well) on a sterile coverslip in a culture 24 well-plate. After $24 \mathrm{~h}$, cells were incubated in a basal growth medium for $48 \mathrm{~h}$ at $37{ }^{\circ} \mathrm{C}$ alone (control) or in the presence of drug-loaded $\alpha$-elastin-g-PLGA nanoparticles dispersed in DMEM. As a comparison, cells were also incubated in a basal growth medium in the presence of free drug dissolved in DMSO. The concentration of free dexamethasone was $50 \mu \mathrm{g} /$ $\mathrm{mL}$, and the amount of drug-loaded $\alpha$-elastin-g-PLGA nanoparticles was $450 \mu \mathrm{g} / \mathrm{mL}$ (this value corresponds to a drug concentration of $50 \mu \mathrm{g} / \mathrm{mL})$. A blank of $\alpha$-elastin-g-PLGA nanoparticles without drug was also assayed by using a concentration of $450 \mu \mathrm{g} / \mathrm{mL}$. The viability of each sample was evaluated using the 3-(4,5-dimethylthiazol-2-yl)-5-(3carboxymethoxyphenyl)-2- (4-sulfophenyl)-2H-tetrazolium, inner salt (MTS) assay (Cell Counting Kit; Sigma). After 3 $\mathrm{h}$, the absorbance of each sample was read at $490 \mathrm{~nm}$ in a 

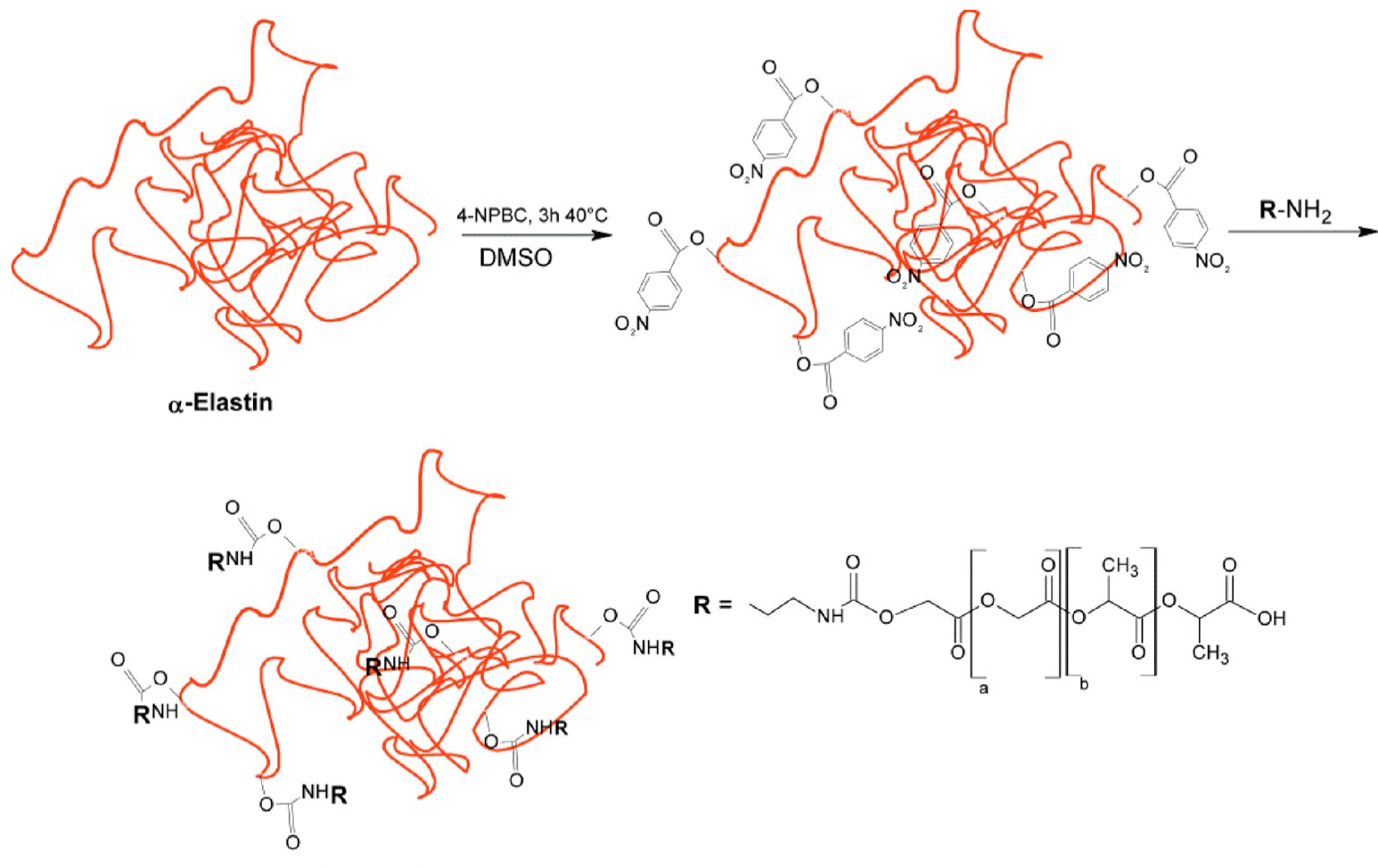

$\alpha$-Elastin-g-PLGA

Figure 1. Schematic representation of activation of $\alpha$-elastin with 4-NPBC and grafting of PLGA-EDA to obtain $\alpha$-elastin-g-PLGA copolymer.

GloMax-Multi Detection System (Promega). Each experiment was performed in triplicate.

\section{RESULTS AND DISCUSSION}

Despite a wide application of native elastin in regenerative medicine, ${ }^{18}$ its use for the production of nanoparticles for drug

Table 1. Grafting Degree in PLGA (GD PLGA mol \%) for $\alpha$ Elastin-g-PLGA Copolymers Obtained by Using Different Weight Ratios between PLGA-EDA and $\alpha$-Elastin (Mean Value \pm Standard Deviation, $n=3$ )

$\begin{array}{cc}\text { weight ratio PLGA-EDA/ } \alpha \text {-elastin } & \mathrm{GD}_{\mathrm{PLGA}}(\mathrm{mol} \%) \\ 2 \text { (sample a) } & 1.28 \pm 0.04 \\ 4 \text { (sample b) } & 1.38 \pm 0.02 \\ 6 \text { (sample c) } & 1.32 \pm 0.02\end{array}$

Table 2. Values of Drug Loading (\% w/w) and Loading Efficacy $(\% \mathrm{w} / \mathrm{w})$ in $\boldsymbol{\alpha}$-Elastin-g-PLGA Nanoparticles Prepared by Using Different Concentrations of Sample a (Mean Value \pm Standard Deviation, $n=3$ )

$\begin{array}{ccc}\begin{array}{c}\text { concentration of } \alpha \text {-elastin-g-PLGA } \\ (\text { sample a) }(\mathrm{mg} / \mathrm{mL})\end{array} & \begin{array}{c}\text { drug loading } \\ (\% \mathrm{w} / \mathrm{w})\end{array} & \begin{array}{c}\text { loading efficacy } \\ (\% \mathrm{w} / \mathrm{w})\end{array} \\ 2.5 & 1.0 \pm 0.2 & 3 \pm 0.1 \\ 5 & 3.0 \pm 0.3 & 9 \pm 0.3 \\ 7.5 & 10.0 \pm 0.2 & 30 \pm 0.2 \\ 15 & 13.0 \pm 0.1 & 39 \pm 0.2\end{array}$

release is limited by its poor solubility in several media. The acidic hydrolysis of desmosine bridges in native elastin allows the production of $\alpha$-elastin soluble both in aqueous and organic media. ${ }^{19}$ Recently, $\alpha$-elastin has been employed for the production of nanoparticles by high temperature coacervation and cross-linking through $\gamma$ irradiation. ${ }^{20}$ On the other hand, it
Table 3. Values of Mean Diameter (MD), Polydispersity Index (PDI), and Zeta Potential (Pz) of Unloaded and DrugLoaded $\alpha$-Elastin-g-PLGA Nanoparticles in Bidistilled Water, DPBS pH 7.4 and DMEM pH 7.4

\begin{tabular}{clccc}
$\begin{array}{c}\alpha \text {-elastin-g-PLGA } \\
\text { nanoparticles }\end{array}$ & external medium & $\begin{array}{c}\text { MD } \\
(\mathrm{nm})\end{array}$ & PDI & \multicolumn{1}{c}{$\mathrm{Pz}$} \\
\hline drug unloaded & bidistilled water & 198.8 & 0.356 & -20.3 \\
& DPBS pH 7.4 & 204.6 & 0.264 & -9.9 \\
& DMEM pH 7.4 & 184.8 & 0.307 & -9.2 \\
drug loaded & bidistilled water & 224.9 & 0.390 & -22.8 \\
& DPBS pH 7.4 & 220.7 & 0.322 & -10.8 \\
& DMEM pH 7.4 & 221.0 & 0.382 & -9.5 \\
\hline
\end{tabular}

is well-known that nanocarriers are useful to improve bioavailability of poorly water-soluble drugs; ${ }^{21}$ therefore, the grafting of a hydrophobic moiety on the $\alpha$-elastin chain could be exploited to produce appropriate nanoparticles able to entrap lipophilic drugs, such as dexamethasone dipropionate. For this reason, in this work, PLGA with a $M_{\mathrm{w}}$ value of about $3000 \mathrm{Da}$ has been chosen as a hydrophobic moiety and grafted to $\alpha$-elastin through a simple and reproducible chemistry, even if $\alpha$-elastin has few reactive groups (more than $80 \%$ of aminoacids are alanin, valine, proline, and glycine) ${ }^{16}$

4-NPBC has been chosen as an activator of hydroxyl groups in $\alpha$-elastin (see Figure 1), and about $1.8 \mathrm{~mol} \%$ of total aminoacids have been activated as evaluated by UV analysis (see the Experimental Section). This amount corresponds to about $50 \mathrm{~mol} \%$ of hydroxyl groups containing aminoacids. At the same time, the reaction between PLGA activated by 4nitrophenyl chloroformate and ethylenediamine (EDA) has been performed to obtain PLGA-EDA derivative. Then, the reaction between PLGA-EDA and the activated $\alpha$-elastin has been performed in anhydrous DMSO, by using three different 

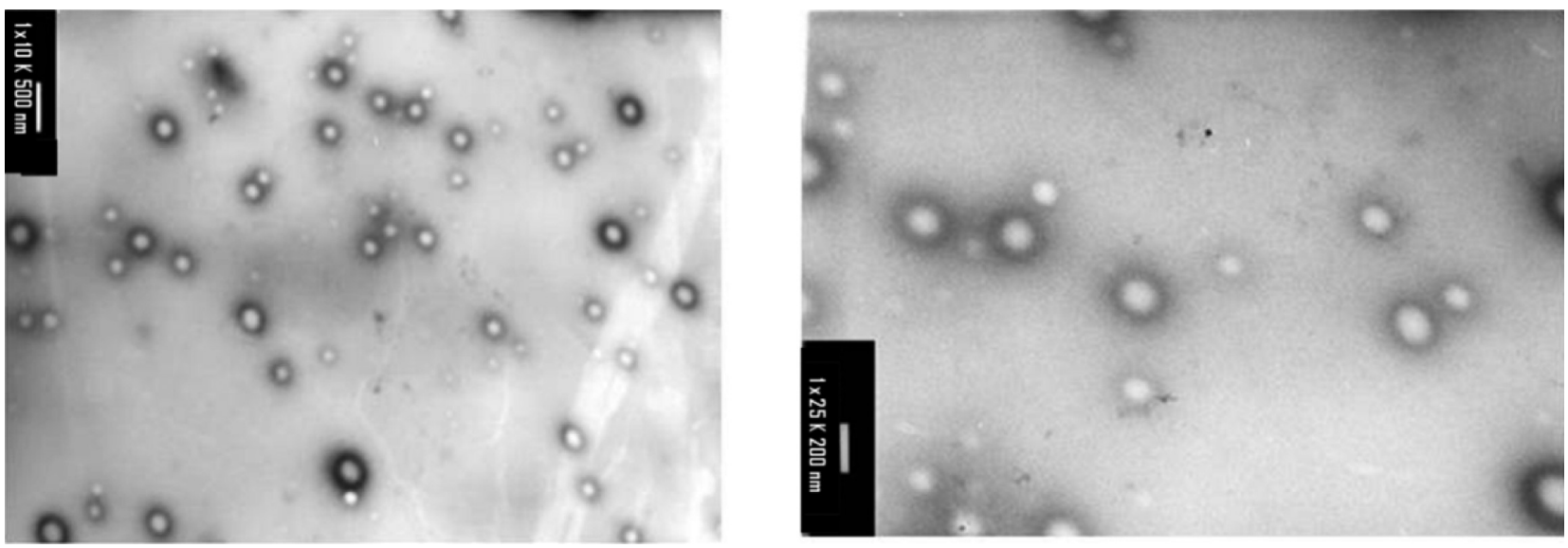

Figure 2. TEM pictures of drug unloaded $\alpha$-elastin-g-PLGA nanoparticles obtained at 10k (left) and 25k (right).

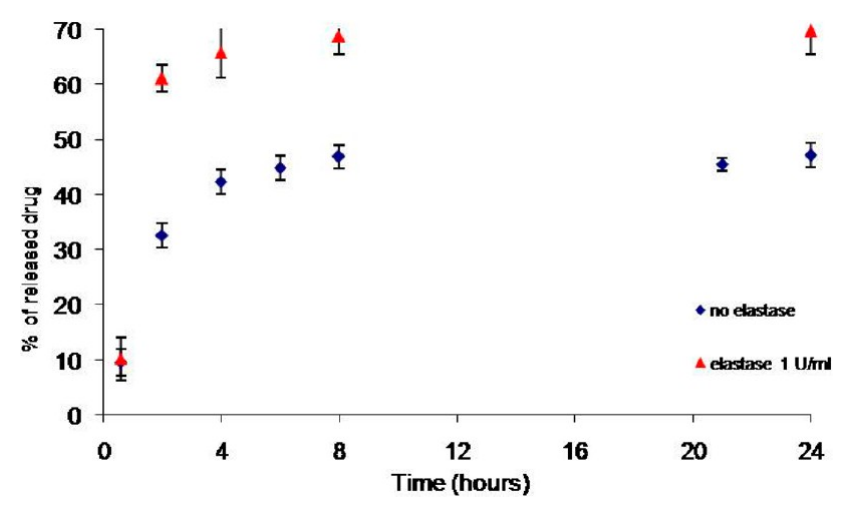

Figure 3. In vitro release profiles of dexamethasone dipropionate from $\alpha$-elastin-g-PLGA nanoparticles in DPBS pH 7.4 at $37^{\circ} \mathrm{C}$. Experiments were performed in the absence $(\checkmark)$ or in the presence $(\boldsymbol{A})$ of elastase $(1 \mathrm{U} / \mathrm{mL})$. weight ratios of PLGA-EDA/ $\alpha$-elastin $(2,4$, or 6) in order to synthesize the corresponding $\alpha$-elastin-g-PLGA copolymer (sample a, b, or c) (see Figure 1).

${ }^{1} \mathrm{H}$ NMR and FTIR analyses performed on each $\alpha$-elastin-gPLGA copolymer have confirmed the success of the grafting procedure. Table 1 reports the amount of PLGA grafted to $\alpha$ elastin, calculated by ${ }^{1} \mathrm{H}$ NMR analysis for all copolymers (see the Experimental Section).

It is evident that the grafting degree in PLGA does not change significantly by increasing the weight ratio of PLGA$\mathrm{EDA} / \alpha$-elastin, and a mean value equal to about $1.3 \mathrm{~mol} \%$ has been obtained. This amount corresponds to the functionalization of about $40 \%$ of total free hydroxyl groups in $\alpha$-elastin, and this means that the $80 \%$ of aminoacids activated with $4-\mathrm{NPBC}$ (that are $1.8 \mathrm{~mol} \%$ as calculated by colorimetric assay) efficiently reacted with PLGA-EDA, thus confirming the success of the grafting reaction of PLGA-EDA on the $\alpha$-elastin chain.
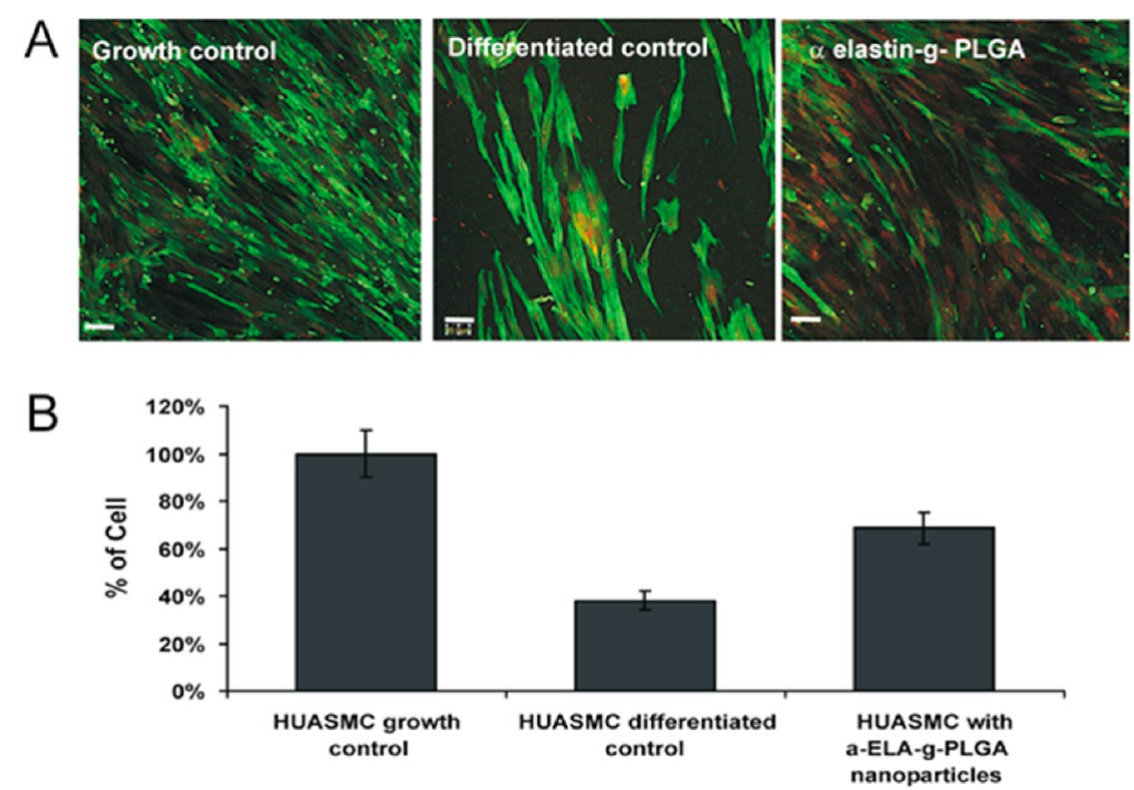

Figure 4. (A) Morphological analysis: HUASMCs incubated in a basal growth medium alone (growth control) or in the presence of $\alpha$-elastin-gPLGA nanoparticles, without drug. Cells incubated with differentiating growth medium have been used as a differentiated control. Cells were stained with phalloidin-FITC (green) and propidium iodide (red) and observed by a confocal microscope. (B) \% of cells (as amount of red fluorescent nuclei) for each culture condition. The analysis was carried out by Image J64 software. 

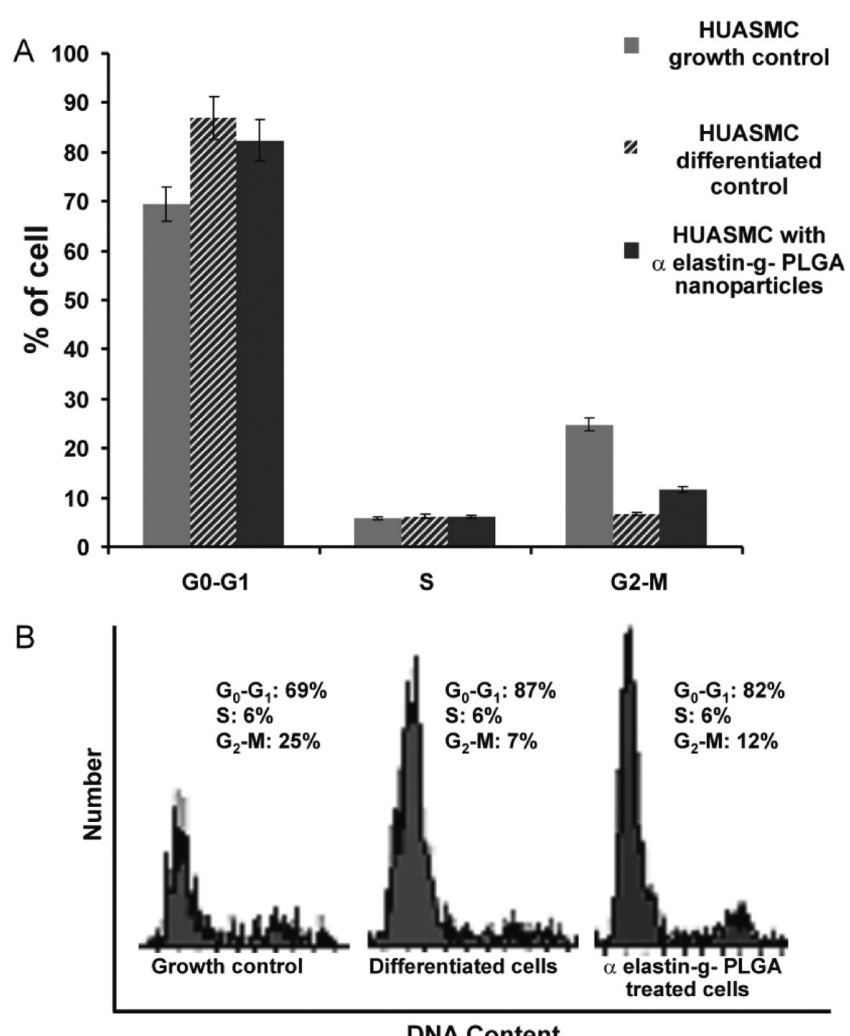

Figure 5. Flow cytometric profile of HUASMCs incubated in a basal growth medium alone (growth control) or in the presence of $\alpha$-elasting-PLGA nanoparticles, without drug. Cells incubated with differentiating growth medium have been used as a differentiated control. The histograms report the \% of cells in each phase of the cell cycle.

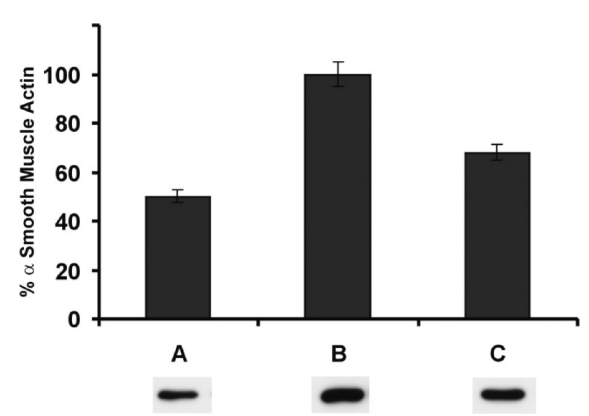

Figure 6. Western blotting analysis for $\alpha \mathrm{SM}$ actin expression from HUASMCs incubated in a basal growth medium alone (growth control, A) or in the presence of $\alpha$-elastin-g-PLGA nanoparticles, without drug (C). Cells incubated with differentiating growth medium have been used as a differentiated control (B). The histograms report the densitometric evaluation of each line, determined by Image J64 software. The values were expressed as \% of $\alpha$ SM actin for each sample.

All $\alpha$-elastin-g-PLGA copolymers were not soluble in water but very soluble in DMSO; however, considering the similar grafting degree in PLGA, only one copolymer (sample a) has been chosen for the production of nanoparticles. In particular, as reported in the Experimental Section, $\alpha$-elastin-g-PLGA nanoparticles have been prepared by the nanoprecipitation technique using a copolymer (sample a) concentration ranging from 2.5 to $15 \mathrm{mg} / \mathrm{mL}$ in DMSO and distilled water has been used as a precipitating medium. Drug-loaded nanoparticles have been prepared by dissolving dexamethasone dipropionate in
DMSO in an amount corresponding to 0.5 -fold the weight of copolymer.

As shown in Table 2, both drug loading and loading efficacy increase with copolymer concentration, reaching a value of 13 and $39 \% \mathrm{w} / \mathrm{w}$, respectively, for a concentration of $15 \mathrm{mg} / \mathrm{mL}$. However, at this concentration, drug-loaded nanoparticles were not dispersible in aqueous medium; on the contrary, nanoparticles obtained with a copolymer concentration of $7.5 \mathrm{mg} /$ $\mathrm{mL}$ give a fine aqueous dispersion. For this reason, nanoparticles having a drug loading of $10 \% \mathrm{w} / \mathrm{w}$ and a loading efficacy of $30 \% \mathrm{w} / \mathrm{w}$ have been used for further characterization.

Table 3 reports the values of mean diameter and zeta potential of these nanoparticles determined in different media by dynamic light scattering measurements.

For each sample, no significant difference has been found by changing the external medium. The presence of drug causes an increase of about $10 \%$ in nanoparticle diameter, but it does not affect the value of the zeta potential, thus indicating that dexamethasone dipropionate is above all entrapped inside the nanoparticle and not on the surface. TEM analysis has confirmed dynamic light scattering data, showing nanoparticles of about $200 \mathrm{~nm}$ with a narrow size distribution. Figure 2 reports, as an example, TEM images of drug unloaded nanoparticles.

The release profile reported in Figure 3 shows that, in the absence of enzyme, a plateau is obtained after $8 \mathrm{~h}$ and only about $40 \%$ of drug is released. The presence of elastase $(1 \mathrm{U} /$ $\mathrm{mL}$ ) causes a pronounced increase in the amount of released drug that, after $4 \mathrm{~h}$, is about $70 \%$ with respect to the total amount. This result demonstrates that $\alpha$-elastin even if grafted to PLGA maintains its susceptibility toward elastase that is able to hydrolyze its chains, thus allowing a faster drug release. However, after $24 \mathrm{~h}$, a significant amount of drug remains still entrapped into the nanoparticles, both in the absence and in the presence of elastase. Unfortunately, after $24 \mathrm{~h}$, dexamethasone dipropionate released in DPBS pH 7.4 undergoes a significant hydrolysis, giving rise to dexamethasone monopropionate and dexamethasone, thus making the analysis of release profile for longer times difficult. However, in order to evaluate if $\alpha$-elasting-PLGA nanoparticles are able to prolong the drug release, after 10 days, nanoparticle dispersion was recovered from the dialysis bag and freeze-dried to determine the amount of dexamethasone dipropionate remaining in the nanoparticles. This amount resulted in being the $10 \% \mathrm{w} / \mathrm{w}$ for nanoparticles treated with elastase and $25 \% \mathrm{w} / \mathrm{w}$ for nanoparticles in the absence of elastase. The obtained result suggests that $\alpha$-elastin-g-PLGA nanoparticles are able to release in a prolonged way (for more than 10 days) a poorly soluble drug, such as dexamethasone dipropionate.

As reported in the Introduction, both elastin and dexamethasone have an inhibitory effect on SMC proliferation, even if with a different mechanism. Therefore, with the aim of differentiating between drug-loaded nanoparticles and nondrug-loaded nanoparticles, different biological assays have been performed, i.e., (1) morphological, flow cytofluorimetric, and Western blotting analyses to evaluate the role of $\alpha$-elastin in $\alpha$ elastin-g-PLGA nanoparticles in the absence of drug and (2) a MTS assay to evaluate the antiproliferative effect of dexamethasone dipropionate loaded into $\alpha$-elastin-g-PLGA nanoparticles in comparison with empty $\alpha$-elastin-g-PLGA nanoparticles and free dexamethasone dipropionate. 


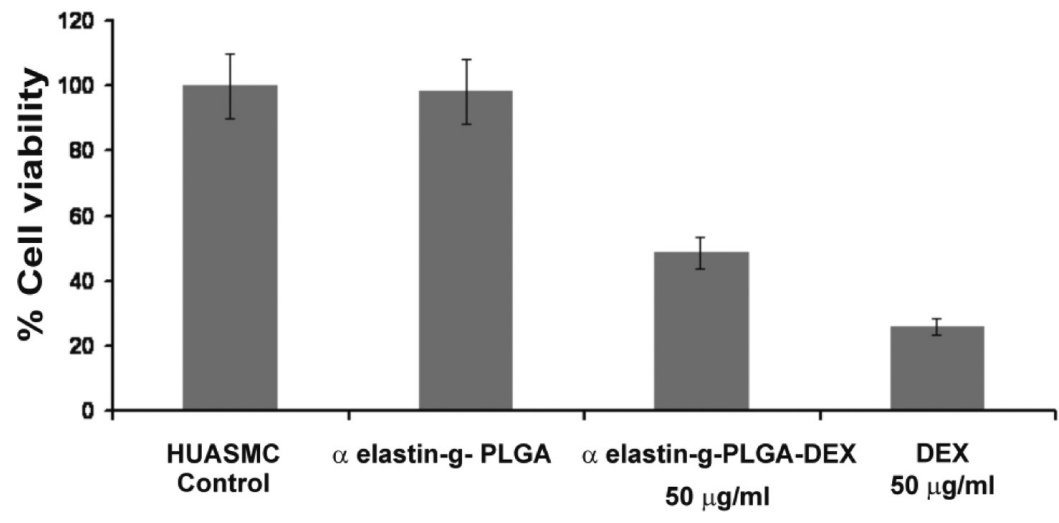

Figure 7. Viability of HUASMCs determined by MTS assay after their incubation with basal growth medium alone (control) or in the presence of $\alpha$ elastin-g-PLGA nanoparticles without drug (blank), drug-loaded $\alpha$-elastin-g-PLGA nanoparticles $(450 \mu \mathrm{g} / \mathrm{mL})$, or free drug (DEX, $50 \mu \mathrm{g} / \mathrm{mL})$. The amount of drug-loaded $\alpha$-elastin-g-PLGA nanoparticles $(450 \mu \mathrm{g} / \mathrm{mL})$ corresponds to a DEX concentration of $50 \mu \mathrm{g} / \mathrm{mL}$.

In particular, by morphological analysis, the potential of $\alpha$ elastin-g-PLGA nanoparticles to stimulate the differentiation toward the contractile phenotype of HUASMCs during the in vitro culture has been evaluated. Cells have been incubated with basal growth medium alone (growth control) or in the presence of $\alpha$-elastin-g-PLGA nanoparticles. Cells incubated with differentiating growth medium have been used as a differentiated control (see the Experimental Section). In agreement with data reported in the literature, the morphological analysis (Figure 4A) evidenced that cells treated with $\alpha$-elastin-g-PLGA nanoparticles show in their cytoplasm a highly organized network of actin stress fibers, a hallmark of mature contractile vascular smooth muscle cells, like the differentiated control.

An image analysis has been performed using Image J64 software to evaluate the number of cells for each colture condition. In particular, the $\%$ of red fluorescent nuclei for each sample was quantified and reported in Figure 4B. It is evident that the \% of HUASMCs decreases with the following order: control cells (considered as $100 \%$ of proliferation) $>$ cells treated with nanoparticles $(69 \%)>$ differentiated control (38\%). This result suggests that proliferation of cells treated with $\alpha$-elastin-g-PLGA nanoparticles (without drug) decreases due to the gradual differentiation toward the contractile phenotype.

To confirm these data, flow cytofluorimetric analysis has also been performed. As it is possible to observe in Figure 5, for HUASMCs treated with $\alpha$-elastin-g-PLGA nanoparticles (without drug), like for differentiated control, the \% of cells in phase G0-G1 is greater than growth control, thus confirming the differentiation toward a contractile phenotype due to the presence of $\alpha$-elastin-g-PLGA nanoparticles.

Moreover, by a Western blotting analysis, the amount of $\alpha$ smooth muscle actin ( $\alpha$ SM actin) has been determined, with this protein being a specific marker of SMC differentiation. ${ }^{22}$ As shown in Figure 6, the $\%$ of $\alpha$ SM actin in cells treated both with $\alpha$-elastin-g-PLGA nanoparticles and with differentiating growth medium is higher than growth control, thus confirming again that the presence of $\alpha$-elastin-g-PLGA nanoparticles, without drug, promotes the differentiation toward the contractile phenotype.

However it is appropriate to relate the percentage of expression of $\alpha \mathrm{SM}$ actin with the mean area of the cells cultured under different conditions. Indeed, it is important to specify that, for sample A (growth control) of Figure 6, the $\alpha$ $\mathrm{SM}$ actin expression (basal level) is due to a high number of cells present in the control. On the contrary, the $\alpha \mathrm{SM}$ actin expression for sample C (HUASMCs treated with $\alpha$-elastin-gPLGA nanoparticles) is due to a lower number of cells (with a greater mean area) having a differentiated contractile phenotype.

Finally, a MTS assay has been performed to evaluate the antiproliferative effect of dexamethasone dipropionate loaded into nanoparticles. HUAMCs incubated in basal growth medium were used as a control, whereas $\alpha$-elastin-g-PLGA nanoparticles without drug were used as a blank. Results have been compared with those obtained by using free dexamethasone dipropionate dissolved in DMSO.

As shown in Figure 7, viability of HUASMCs incubated for $48 \mathrm{~h}$ in the presence of $\alpha$-elastin-g-PLGA nanoparticles without drug (blank) is equal to that of cells treated with basal growth medium alone. On the contrary, drug-loaded nanoparticles cause a pronounced decrease in cell viability like free dexamethasone dipropionate. The obtained results demonstrate that, according to the different action mechanism of $\alpha$-elastin and dexamethasone, as mentioned above, MTS assay allows one to evaluate only the antiproliferative effect of drug entrapped into nanoparticles.

To sum up, biological assays differentiate between drugloaded and non-drug-loaded nanoparticles, thus demonstrating the benefit due to the combination of $\alpha$-elastin-g-PLGA and dexamethasone dipropionate for a potential treatment of restenosis.

\section{CONCLUSIONS}

A simple and reproducible grafting reaction has been described to obtain an $\alpha$-elastin-g-PLGA copolymer employed for the production of nanoparticles potentially useful to prevent restenosis. In particular, nanoparticles with a mean diameter of $200 \mathrm{~nm}$ have been produced and successfully employed to entrap dexamethasone dipropionate, a known drug that inhibits proliferation of vascular smooth muscle cells (VSMCs). Thanks to the activity of $\alpha$-elastin in regulating smooth muscle cell phenotype, $\alpha$-elastin-g-PLGA nanoparticles alone are able to stimulate the differentiation of human umbilical artery smooth muscle cells toward the contractile phenotype, as evidenced by different biological studies like immunofluorescence, flow cytofluorimetric, and western blotting analyses. Moreover, drug-loaded nanoparticles exhibit in aqueous medium a good antiproliferative effect comparable to that of free drug dissolved in organic medium. Therefore, $\alpha$-elastin-g-PLGA nanoparticles 
show an intrinsic ability to promote the differentiation toward the contractile phenotype combined with the antiproliferative effect of dexamethasone dipropionate. In addition, release studies have shown that $\alpha$-elastin-g-PLGA nanoparticles act as a drug reservoir, thus allowing a prolonged release in aqueous medium without the use of organic solvent. The obtained results can be considered as positive preliminary data for a further design of innovative devices for restenosis treatment.

\section{AUTHOR INFORMATION}

\section{Corresponding Author}

*E-mail: giovanna.pitarresi@unipa.it. Phone: 0039091 23891954. Fax: 003909123891960.

\section{Author Contributions}

The manuscript has been written thanks to the contribution of all authors that have approved its final version.

\section{Notes}

The authors declare no competing financial interest.

\section{ACKNOWLEDGMENTS}

The authors thank M.I.U.R. for financial support.

\section{REFERENCES}

(1) Karnik, S. K.; Brooke, B. S.; Bayes-Genis, A.; Sorensen, L.; Wythe, J. D.; Schwartz, R. S.; Keating, M. T.; Li, D. Y. A critical role for elastin signaling in vascular morphogenesis and disease. Development 2003, 130, 411-423.

(2) Chan-Park, M. B.; Shen, J. Y.; Cao, Y.; Xiong, Y.; Liu, Y.; Rayatpisheh, S.; Kang, G. C.; Greisler, H. P. Biomimetic control of vascular smooth muscle cell morphology and phenotype for functional tissue-engineered small-diameter blood vessels. J. Biomed. Mater. Res., Part A 2009, 88, 1104-1121.

(3) Reil, T. D.; Sarkar, R.; Kashiap, V. S.; Sarkar, M.; Gelabert, H. A. Dexamethasone suppresses Vascular Smooth Muscle Cell proliferation. J. Surg. Res. 1999, 85, 109-114.

(4) Liu, K.; Cao, G.; Zhang, X.; Liu, R.; Zou, W.; Wu, S. Pretreatment with intraluminal rapamycin nanoparticle perfusion inhibits neointimal hyperplasia in a rabbit vein graft model. Int. J. Nanomed. 2010, 5, 853860.

(5) Hunter, W. L. Drug-eluting stents: Beyond the hyperbole. Adv. Drug Delivery Rev. 2006, 58, 347-349.

(6) Acharya, G.; Park, K. Mechanism of controlled drug release from drug-eluting stents. Adv. Drug. Delivery Rev. 2006, 58, 387-401.

(7) Labhasetwar, V.; Song, C.; Levy, R. J. Nanoparticle drug delivery system for restenosis. Adv. Drug. Delivery Rev. 1997, 24, 63-85.

(8) Kavanagh, C. A.; Rochev, Y. A.; Gallagher, W. M.; Dawson, K. A.; Keenan, A. K. Local drug delivery in restenosis injury: thermoresponsive co-polymers as potential drug delivery systems. Pharmacol. Ther. 2004, 102, 1-15.

(9) Hedin, U.; Roy, J.; Tran, P. K. Control of smooth muscle cell proliferation in vascular disease. Curr. Opin. Lipidol. 2004, 15, 559565.

(10) Schwartz, R. S.; Huber, K. C.; Murphy, J. G.; Edwards, W. D.; Camrud, A. R.; Vlietstra, R. E.; Holmes, D. R. J. Restenosis and the proportional neointimal response to coronary artery injury: results in a porcine model. J. Am. Coll. Cardiol. 1992, 19, 267-274.

(11) Goldsmith, A. M.; Hershenson, M. B.; Wolbert, M. P.; Bentley, J. K. Regulation of airway smooth muscle $\alpha$-actin expression by glucocorticoids. Am. J. Physiol.: Lung Cell. Mol. Physiol. 2007, 292, 99106.

(12) Zhou, Z. X.; Zhang, B. G.; Zhang, H.; Huang, X. Z.; Hu, Y. L.; Sun, L.; Wang, X. M.; Zhang, J. W. Drug packaging and delivery using perfluorocarbon nanoparticles for targeted inhibition of vascular smooth muscle cells. Acta. Pharmacol. Sin. 2009, 30, 1577-1515.
(13) Partridge, S. M.; Davis, H. F.; Adair, G. S. The chemistry of connective tissues. 2. Soluble proteins derived from partial hydrolysis of elastin. Biochem. J. 1955, 61, 11-21.

(14) Choi, J. S.; Leong, K. W.; Sang, Y. Y. In vivo wound healing of diabetic ulcers using electrospun nanofibers immobilized with human epidermal growth factor. Biomaterials 2008, 29, 587-596.

(15) Palumbo, F. S.; Pitarresi, G.; Fiorica, C.; Rigogliuso, S.; Ghersi, G.; Giammona, G. Chemical hydrogels based on a hyaluronic acidgraft- $\alpha$-elastin derivative as potential scaffolds for tissue engineering. Mater. Sci. Eng., C 2013, 33, 2541-2549.

(16) Leach, J. B.; Wolinsky, J. B.; Stone, P. J.; Wong, J. Y. Crosslinked alpha-elastin biomaterials: towards a processable elastin mimetic scaffold. Acta Biomater. 2005, 1, 155-164.

(17) Deroanne, C. F.; Lapiere, C. M.; Nusgens, B. V. In vitro tubulogenesis of endothelial cells by relaxation of the coupling extracellular matrix-cytoskeleton. Cardiovasc. Res. 2001, 49, 647-658.

(18) Daamen, W. F.; Veerkamp, J. H.; van Hest, J. C. M.; van Kuppervelt, T. H. Elastin as biomaterial for tissue engineering. Biomaterials 2007, 28, 4378-4398.

(19) Annabi, N.; Mithieux, S. M.; Boughton, E. A.; Ruys, A. J.; Weiss, A. S.; Dehgani, F. Synthesis of highly porous crosslinked elastin hydrogels and their interaction with fibroblasts in vitro. Biomaterials 2009, 30, 4550-4557.

(20) Fujimoto, M.; Takeda, M.; Okamoto, K.; Furuta, M. Effect of gamma irradiation dose on the fabrication of $\alpha$-elastin nanoparticles by gamma-ray crosslinking. Radiat. Phys. Chem. 2011, 80, 142-144.

(21) Torchilin, V. P. Multifunctional nanocarriers. Adv. Drug. Delivery Rev. 2006, 58, 1532-1555.

(22) Skalli, O.; Pelte, M. F.; Peclet, M. C.; Gabbiani, G.; Gugliotta, P.; Bussolati, G.; Ravazzola, M.; Orci, L. Alpha-smooth muscle actin, a differentiation marker of smooth muscle cells, is present in microfilamentous bundles of pericytes. J. Histochem. Cytochem. 1989, $37,315-321$. 